\title{
Existence, uniqueness and stability of the solution to neutral stochastic functional differential equations with infinite delay under non-Lipschitz conditions
}

Fengying Wei* and Yuhua Cai

"Correspondence:

weifengying@fzu.edu.cn College of Mathematics and Computer Science, Fuzhou University, Fuzhou, 350116 P.R. China

\section{算 Springer}

\begin{abstract}
Choosing space $C_{g}$ as the phase space, the existence, uniqueness and stability of the solution to neutral stochastic functional differential equations with infinite delay (short for INSFDEs) are studied in this paper. Under non-Lipschitz condition, weakened linear growth condition and contractive condition, the existence-and-uniqueness theorem of the solution to INSFDEs by means of the Picard iteration, Doob's martingale inequalities, Gronwall's inequality and Bihari's inequality is obtained. Furthermore, the continuous dependence of the solutions on the initial value to INSFDEs are derived.
\end{abstract}

MSC: $65 \mathrm{C} 30 ; 60 \mathrm{H} 10$

Keywords: neutral stochastic functional differential equations; infinite delay; phase space $C_{g}$; non-Lipschitz condition; existence-and-uniqueness theorem; stability

\section{Introduction}

With the development of industrial technology, just using ordinary differential equations to describe the systems with the phenomenon of time delay is not very ideal in real life. So, we cannot ignore the impact of system-related time delay. Namely, the information provided by the historical state of the system is the necessary data for the development to the current state. Such a system which depends on the historical information is called functional differential equations [1]. In general, functional differential equations are widely used in the fields of biological engineering, chemistry and chemical engineering etc. For functional differential equations with infinite delay, the selection of phase space plays a key role in solving specific problems [2]. In 1978, Hale et al. [3], Schumacher [4, 5] built a foundation of phase space axiomatic, respectively. In 1980, Corduneana et al. [6] summarized the theory of functional differential equations with infinite delay. Almost at the same time, Wang et al. built $C_{h}$ phase space and Burton constructed $C_{g}$ phase space, respectively [2]; these two kinds of phase spaces are different, and they are convenient for establishing the qualitative properties of solutions to functional differential equations with infinite delay.

In recent years, the existence-and-uniqueness theorem, stability and other related properties of stochastic functional differential equations have attracted great attention (see [7-9] and references therein for details). In 2006, Wei [10] studied the existence-and-

C 2013 Wei and Cai; licensee Springer. This is an Open Access article distributed under the terms of the Creative Commons Attribution License (http://creativecommons.org/licenses/by/2.0), which permits unrestricted use, distribution, and reproduction in any medium, provided the original work is properly cited. 
uniqueness theorem of solutions to stochastic functional differential equations with infinite delay (ISFDEs in short) at phase space $B C\left((-\infty, 0] ; \mathrm{R}^{d}\right), C_{g}$ and $C_{h}$, respectively. Furthermore, she obtained the estimate for the error between the approximate solution and the accurate one to ISFDEs, which generalized Mao's conclusions [7]. In 2007, under uniform Lipschitz condition and local Lipschitz respectively, Wei et al. [11] considered ISFDEs at phase space $B C\left((-\infty, 0] ; \mathrm{R}^{d}\right)$, they obtained the existence-and-uniqueness theorem and the moment estimate of solutions. In 2008, Xu et al. [12] established the existence, uniqueness and continuity of the local solutions to ISFDEs by means of Driver theorem and Picard iteration. In 2010, Yue et al. [13] investigated the existence, uniqueness and stability of solutions to ISFDEs under non-Lipschitz condition. Some authors introduced the theory of delayed stochastic functional differential equations into a neural network. For instance, Su et al. [14] considered an $n$-dimensional stochastic neural network with infinite delay (ISNN in short) in 2011. They obtained some sufficient conditions for stochastic stability, stochastic asymptotical stability and global stochastic asymptotic stability under some basic assumptions. Moreover, the stochastic stability of ISNN would not be changed when the environmental noises were small. In 2013, Zhang et al. [15] obtained sufficient criteria for stability and boundedness of solutions by the Lyapunov-Krasovskii functional approach to stochastic Volterra integrodifferential equations with infinite delay.

Stochastic system depends not only on the current state and a period of past state, but also on the rate of change for the past state, the systems are said to be neutral stochastic functional differential equations (NSFDEs in short) [7]. In 2000, under the classic Lipschitz condition, Mao $[7,16]$ discussed the existence-and-uniqueness theorem, the $L^{p}$-exponential estimates, exponential stability and almost sure exponential stability for a solution to NSFDEs. For neutral stochastic functional differential equations with infinite delay (INSFDEs in short), in 2008, Zhou et al. [17] established the existence and uniqueness theorem of solutions to INSFDEs under uniform Lipschitz condition. In 2009, under non-Lipschitz condition, Ren et al. [18] obtained the existence-and-uniqueness theorem and continuous dependence of solutions to INSFDEs by means of Bihari's inequality at phase space $B C\left((-\infty, 0] ; \mathrm{R}^{d}\right)$. In 2010, Xu et al. [19] studied the existence and uniqueness of the solution to INSFDEs under Lipschitz condition through establishing a Banach space $\left(\|\cdot\|_{M^{2}}, M^{2}\left((-\infty, T] ; \mathrm{R}^{d}\right)\right)$. Chen [20] investigated the existence and uniqueness of solutions to INSFDEs under uniform Lipschitz condition, linear growth condition and contractive condition at phase space $B C\left((-\infty, 0] ; \mathrm{R}^{d}\right)$ by constructing a new iterative by the fixed point theorem. Then, he got the moment estimate of solutions and the estimate for the error between the approximate solution and the accurate solution.

Motivated by the work [18], we choose $C_{g}$ as our phase space in this paper, the existence-and-uniqueness theorem of the solutions to INSFDEs will be obtained under non-Lipschitz condition, weakened liner growth condition and contractive condition. Furthermore, the continuous dependence of solutions on the initial value is given.

\section{Preliminary}

Let $\mathrm{R}^{-}=(-\infty, 0], \mathrm{R}^{+}=[0,+\infty), C=C\left(\mathrm{R}^{-}, \mathrm{R}^{n}\right)$ denote the family of continuous functions from $\mathrm{R}^{-}$to $\mathrm{R}^{n}$, let $g: \mathrm{R}^{-} \rightarrow[1, \infty)$ be a continuous and non-increasing function such that $g(0)=1, g(-\infty):=\lim _{x \rightarrow-\infty} g(x)=+\infty$. Define

$$
C_{g}=\left\{\psi \in C: \frac{\psi}{g} \text { be uniform continuous on } \mathrm{R}^{-} \text {and } \sup _{s \leq 0} \frac{|\psi(s)|}{g(s)}<\infty\right\} .
$$


For any $\psi \in C_{g}$, the norm be defined as $|\psi|_{g}=\sup _{s \leq 0} \frac{|\psi(s)|}{g(s)}$. Arion et al. [21] proved that $\left(C_{g},|\cdot|_{g}\right)$ was a Banach space with the norm $|\cdot|_{g}$. We denote by $\mathcal{M}^{2}\left(\left(-\infty, t_{0}\right] ; \mathrm{R}^{d}\right)$ the family of all $\mathcal{F}_{t_{0}}$-measurable, $\mathrm{R}^{d}$-valued process $\varphi(t)=\varphi(t, \omega), t \in\left(-\infty, t_{0}\right]$ such that $E \int_{-\infty}^{t_{0}}|\varphi(t)|^{2} \mathrm{~d} t<\infty$.

Let $(\Omega, \mathcal{F}, \mathrm{P})$, throughout this paper unless otherwise specified, be a complete probability space with a filtration $\left\{\mathcal{F}_{t}\right\}_{t \geq t_{0}}$ satisfying the usual conditions. Assume that $W(t)=$ $\left(W_{1}(t), W_{2}(t), \ldots, W_{m}(t)\right)^{\mathrm{T}}$ is an $m$-dimensional Brownian motion defined on a complete probability space. For $0 \leq t_{0}<T<\infty$, let $f:\left[t_{0}, T\right] \times C_{g} \rightarrow \mathrm{R}^{d}, h:\left[t_{0}, T\right] \times C_{g} \rightarrow \mathrm{R}^{d \times m}$ and $D:\left[t_{0}, T\right] \times C_{g} \rightarrow \mathrm{R}^{d}$ be Borel measurable. Consider the $d$-dimensional neutral stochastic functional differential equation with infinite delay

$$
\mathrm{d}\left(X(t)-D\left(t, X_{t}\right)\right)=f\left(t, X_{t}\right) \mathrm{d} t+h\left(t, X_{t}\right) \mathrm{d} W(t), \quad t_{0} \leq t \leq T,
$$

where $X_{t}=\{X(t+\theta):-\infty<\theta \leq 0\}$ can be regarded as a $C_{g}$-value stochastic process. The initial data of system (1)

$$
X_{t_{0}}=\xi=\{\xi(\theta):-\infty<\theta \leq 0\}
$$

is $\mathcal{F}_{t_{0}}$-measurable, $C_{g}$-valued random variable such that $\xi \in \mathcal{M}^{2}\left(\left(-\infty, t_{0}\right] ; \mathrm{R}^{d}\right)$.

Definition 1 [7] $\mathrm{R}^{d}$-value stochastic process $X(t)$ defined on $-\infty<t \leq T$ is called the solution of (1) with initial data (2) if $X(t)$ has the following properties:

(i) $X(t)$ is continuous and $\{X(t)\}_{t_{0} \leq t \leq T}$ is $\mathcal{F}_{t}$-adapted;

(ii) $\left\{f\left(t, X_{t}\right)\right\} \in \mathcal{L}^{1}\left(\left[t_{0}, T\right] ; \mathrm{R}^{d}\right)$ and $\left\{h\left(t, X_{t}\right)\right\} \in \mathcal{L}^{2}\left(\left[t_{0}, T\right] ; \mathrm{R}^{d \times m}\right)$;

(iii) $X_{t_{0}}=\xi$, for each $t_{0} \leq t \leq T$,

$$
X(t)=\xi(0)+D\left(t, X_{t}\right)-D\left(t_{0}, \xi\right)+\int_{t_{0}}^{t} f\left(s, X_{s}\right) \mathrm{d} s+\int_{t_{0}}^{t} h\left(s, X_{s}\right) \mathrm{d} W(s) \quad \text { a.s. }
$$

$X(t)$ is called a unique solution if any other solution $\bar{X}(t)$ is distinguishable with $X(t)$, that is,

$$
\mathrm{P}\{X(t)=\bar{X}(t) \text { for any }-\infty<t \leq T\}=1
$$

We always assume that (B1)-(B3) are valid in this paper.

(B1) (non-uniform Lipschitz condition) For any $\varphi, \psi \in C_{g}$ and $t \in\left[t_{0}, T\right]$, it follows that

$$
|f(t, \varphi)-f(t, \psi)|^{2} \vee|h(t, \varphi)-h(t, \psi)|^{2} \leq \kappa\left(|\varphi-\psi|_{g}^{2}\right),
$$

where $\kappa(\cdot)$ is a concave continuous nondecreasing function from $\mathrm{R}^{+}$to $\mathrm{R}^{+}$such that $\kappa(0)=0, \kappa(u)>0$ for $u>0$ and $\int_{0^{+}} \frac{1}{\kappa(u)} \mathrm{d} u=\infty$;

(B2) (weakened linear growth condition) For any $t \in\left[t_{0}, T\right]$, it follows that $f(t, 0), h(t, 0) \in L^{2}$ such that $|f(t, 0)|^{2} \vee|h(t, 0)|^{2} \leq K$, where $K>0$ is a constant;

(B3) (contractive condition) There exists a positive constant $K_{0}<\frac{1}{48}$, for any $\varphi, \psi \in C_{g}$ and $t \in\left[t_{0}, T\right]$, it follows that

$$
|D(t, \varphi)-D(t, \psi)|^{2} \leq K_{0}|\varphi-\psi|_{g}^{2} \quad \text { and } \quad|D(t, 0)|^{2} \leq K_{0} .
$$


Definition 2 [18] Solution $X^{\xi}(t)$ of system (1) with initial data (2) is said to be stable in mean square if for all $\varepsilon>0$, there exists $\delta(\varepsilon)>0$. When $E|\xi-\eta|_{g}^{2} \leq \delta(\varepsilon)$, it follows that $E\left|X^{\xi}(t)-Y^{\eta}(t)\right|^{2} \leq \varepsilon$ for all $t \geq t_{0}$, where $Y^{\eta}(t)$ is another solution of system (1) with initial data $\eta \in \mathcal{M}^{2}\left(\left(-\infty, t_{0}\right] ; \mathrm{R}^{d}\right)$.

Lemma 1 (Hölder's inequality) [7] If $\frac{1}{p}+\frac{1}{q}=1$ for any $p, q>1, f \in L^{p}$ and $g \in L^{q}$, then $f g \in L^{1}$ and $\int_{a}^{b} f g \mathrm{~d} x \leq\left(\int_{a}^{b}|f|^{p} \mathrm{~d} x\right)^{\frac{1}{p}}\left(\int_{a}^{b}|g|^{q} \mathrm{~d} x\right)^{\frac{1}{q}}$.

Lemma 2 (Doob's martingale inequality) [7] Let $\{X(t)\}_{t \geq 0}$ be an $\mathrm{R}^{d}$-value martingale and let $[a, b]$ be a bounded interval on $\mathrm{R}^{+}$. If $p>1$ and $X(t) \in L^{p}\left(\Omega, \mathrm{R}^{d}\right)$, then $E\left(\sup _{a \leq t \leq b}|X(t)|^{p}\right) \leq\left(\frac{p}{p-1}\right)^{p} E\left(|X(b)|^{p}\right)$. In particular, $E\left(\sup _{a \leq t \leq b}|X(t)|^{2}\right) \leq 4 E\left(|X(b)|^{2}\right)$ as $p=2$.

Lemma 3 (Gronwall's inequality) [7] Let $\alpha \geq 0, \beta(t) \geq 0$ and let $\varphi(t)$ be a real continuous function on $[a, b]$. If $\varphi(t) \leq \alpha+\int_{a}^{t} \beta(s) \varphi(s) \mathrm{d}$ for all $a \leq t \leq b$, then $\varphi(t) \leq \alpha \exp \int_{a}^{t} \beta(s) \mathrm{d} s$ for all $a \leq t \leq b$.

Lemma 4 [7] If $p \geq 2, h \in \mathcal{M}^{2}\left(\left[t_{0}, T\right] ; \mathrm{R}^{d \times m}\right)$ such that $E \int_{t_{0}}^{T}|h(s)|^{p} \mathrm{~d} s<\infty$, then

$$
E\left|\int_{t_{0}}^{T} h(s) \mathrm{d} W(s)\right|^{p} \leq\left(\frac{p(p-1)}{2}\right)^{\frac{p}{2}}\left(T-t_{0}\right)^{\frac{p-2}{2}} E \int_{t_{0}}^{T}|h(s)|^{p} \mathrm{~d} s .
$$

In particular, $E\left|\int_{t_{0}}^{T} h(s) \mathrm{d} W(s)\right|^{2} \leq E \int_{t_{0}}^{T}|h(s)|^{2} \mathrm{~d} s$ as $p=2$.

Lemma 5 (Bihari's inequality) [7] Let $T \geq 0$ and $u_{0} \geq 0$, let $u(t), v(t)$ be continuous functions on $[0, T]$. Let $\kappa(\cdot): \mathrm{R}^{+} \rightarrow \mathrm{R}^{+}$be a concave continuous and nondecreasing function such that $\kappa(r)>0$ for $r>0$. If $u(t) \leq u_{0}+\int_{t}^{T} v(s) \kappa(u(s)) \mathrm{d}$ for all $0 \leq t \leq T$, then $u(t) \leq G^{-1}\left(G\left(u_{0}\right)+\int_{t}^{T} v(s) \mathrm{d} s\right)$ for all $t \in[0, T]$ such that $G\left(u_{0}\right)+\int_{t}^{T} v(s) \mathrm{d} s \in \operatorname{Dom}\left(G^{-1}\right)$, where $G(r)=\int_{1}^{r} \frac{1}{\kappa(s)} \mathrm{d} s, r \geq 0$ and $G^{-1}$ is the inverse function of $G$.

Lemma 6 [18] Let the assumptions of Lemma 5 hold and $v(t) \geq 0$ for $t \in[0, T]$. If for all $\varepsilon>0$, there exists $t_{1} \geq 0$ such that $\int_{t_{1}}^{T} \nu(s) \mathrm{d} s \leq \int_{u_{0}}^{\varepsilon} \frac{1}{\kappa(s)} \mathrm{d}$ s holds for $0 \leq u_{0} \leq \varepsilon$, then for every $t \in\left[t_{1}, T\right]$, the estimate $u(t) \leq \varepsilon$ holds.

\section{The existence-and-uniqueness theorem}

Let $X^{0}(t)=\xi(0), X_{t_{0}}^{n}=\xi$ for $n=1,2, \ldots$ and all $t_{0} \leq t \leq T$. Define the following Picard sequence

$$
X^{n}(t)=\xi(0)+D\left(t, X_{t}^{n}\right)-D\left(t_{0}, \xi\right)+\int_{t_{0}}^{t} f\left(s, X_{s}^{n-1}\right) \mathrm{d} s+\int_{t_{0}}^{t} h\left(s, X_{s}^{n-1}\right) \mathrm{d} W(s)
$$

If we can give the approximate solutions by means of Picard iteration, then the existenceand-uniqueness theorem of the solutions for INSFDEs at phase space $C_{g}$ can be discussed next.

Theorem 1 If (B1), (B2) and (B3) hold, then there exists a unique solution to system (1) with initial value (2). 
To show Theorem 1, first of all, let us prove two useful lemmas.

Lemma 7 Under the assumptions (B1), (B2) and (B3), for all $n, m \geq 1$, there exist positive constants $\tilde{I}, \tilde{I}_{1}, \tilde{I}_{2}$ such that

$$
\begin{aligned}
& E\left|X^{n}(t)\right|^{2} \leq \tilde{I}, \quad t \in(-\infty, T], \\
& E\left(\sup _{t_{0} \leq s \leq t}\left|X^{n+m}(s)-X^{n}(s)\right|^{2}\right) \\
& \quad \leq \tilde{I}_{1} \int_{t_{0}}^{t} \kappa\left(E\left(\sup _{t_{0} \leq r \leq s}\left|X^{n+m-1}(r)-X^{n-1}(r)\right|^{2}\right)\right) \mathrm{d} s \\
& \quad \leq \tilde{I}_{2}\left(T-t_{0}\right), \quad t \in\left[t_{0}, T\right] .
\end{aligned}
$$

Proof Obviously, $X^{0}(t) \in \mathcal{M}^{2}\left(\left(-\infty, t_{0}\right] ; \mathrm{R}^{d}\right)$. By induction, $X^{n}(t) \in \mathcal{M}^{2}\left(\left(-\infty, t_{0}\right] ; \mathrm{R}^{d}\right)$. In fact, from Hölder's inequality and the elementary inequality $(a+b+c+d)^{2} \leq 4\left(a^{2}+b^{2}+\right.$ $\left.c^{2}+d^{2}\right)$, we have

$$
\begin{aligned}
\left|X^{n}(t)\right|^{2} \leq & 4|\xi(0)|^{2}+4\left|D\left(t, X_{t}^{n}\right)-D\left(t_{0}, X_{t_{0}}^{n}\right)\right|^{2}+4\left|\int_{t_{0}}^{t} f\left(s, X_{s}^{n-1}\right) \mathrm{d} s\right|^{2} \\
& +4\left|\int_{t_{0}}^{t} h\left(s, X_{s}^{n-1}\right) \mathrm{d} W(s)\right|^{2} .
\end{aligned}
$$

Taking the exception on both sides, and by Hölder's inequality and Lemma 4, thus we get

$$
\begin{aligned}
E\left|X^{n}(t)\right|^{2} \leq & 4 E|\xi(0)|^{2}+4 E\left|D\left(t, X_{t}^{n}\right)-D\left(t_{0}, X_{t_{0}}^{n}\right)\right|^{2} \\
& +4 E\left|\int_{t_{0}}^{t} f\left(s, X_{s}^{n-1}\right) \mathrm{d} s\right|^{2}+4 E\left|\int_{t_{0}}^{t} h\left(s, X_{s}^{n-1}\right) \mathrm{d} W(s)\right|^{2} \\
\leq & 4 E\left|D\left(t, X_{t}^{n}\right)-D(t, 0)+D\left(t_{0}, 0\right)-D\left(t_{0}, X_{t_{0}}^{n}\right)+D(t, 0)-D\left(t_{0}, 0\right)\right|^{2} \\
& +8\left(t-t_{0}\right) E \int_{t_{0}}^{t}\left(\left|f\left(s, X_{s}^{n-1}\right)-f(s, 0)\right|^{2}+|f(s, 0)|^{2}\right) \mathrm{d} s \\
& +8 E \int_{t_{0}}^{t}\left(\left|h\left(s, X_{s}^{n-1}\right)-h(s, 0)\right|^{2}+|h(s, 0)|^{2}\right) \mathrm{d} s+4 E|\xi|_{g}^{2} \\
\leq & 4 E|\xi|_{g}^{2}+12 K_{0} E\left|X_{t}^{n}\right|_{g}^{2}+12 K_{0} E\left|X_{t_{0}}^{n}\right|_{g}^{2}+48 K_{0} \\
& +8\left(T-t_{0}+1\right) E \int_{t_{0}}^{t} \kappa\left(\left|X_{s}^{n-1}\right|_{g}^{2}\right) \mathrm{d} s+8\left(T-t_{0}+1\right)\left(T-t_{0}\right) K .
\end{aligned}
$$

Let $z_{0}=48 K_{0}+\left(4+12 K_{0}\right) E|\xi|_{g}^{2}+8\left(T-t_{0}+1\right)\left(T-t_{0}\right) K$. According to the property of a concave function, $\kappa(\cdot)$ always exists maximum on the bounded closed interval $\left[t_{0}, T\right]$, without loss of generality, taking $\max _{t_{0} \leq t \leq T} \kappa\left(X_{t}\right)=\kappa\left(X_{T^{*}}\right)$ as $t=T^{*} \in\left[t_{0}, T\right]$. The line $L$ always lies on the curve $\kappa(\cdot)$, and line $L$ passes through the point $\left(T^{*}, \kappa\left(X_{T^{*}}\right)\right)$ with the slope $\kappa^{\prime}\left(X_{T^{*}}\right)$. Hence, for all $X_{t} \geq 0, t \in\left[t_{0}, T\right]$, it then follows

$$
\kappa\left(X_{t}\right) \leq a+b X_{t}
$$


where $a=\kappa\left(X_{T^{*}}\right)-\kappa^{\prime}\left(X_{T^{*}}\right) X_{T^{*}}$ and $b=\kappa^{\prime}\left(X_{T^{*}}\right)$ are positive constants. Thus, (4) can be simplified as

$$
\begin{aligned}
E\left|X^{n}(t)\right|^{2} & \leq z_{0}+12 K_{0} E\left|X_{t}^{n}\right|_{g}^{2}+8\left(T-t_{0}+1\right) E \int_{t_{0}}^{t}\left(a+b\left|X_{s}^{n-1}\right|_{g}^{2}\right) \mathrm{d} s \\
& \leq z_{1}+12 K_{0} E\left|X_{t}^{n}\right|_{g}^{2}+8 b\left(T-t_{0}+1\right) E \int_{t_{0}}^{t}\left|X_{s}^{n-1}\right|_{g}^{2} \mathrm{~d} s,
\end{aligned}
$$

where $z_{1}=z_{0}+8 a\left(T-t_{0}+1\right)\left(T-t_{0}\right)$.

According to the fact $\left|X_{t}^{n}\right|_{g}=\sup _{v \leq 0} \frac{\left|X_{t}^{n}(v)\right|}{g(v)}=\sup _{v \leq 0} \frac{\left|X^{n}(t+v)\right|}{g(v)}$, one can see that

$$
\sup _{t_{0} \leq s \leq t}\left|X_{s}^{n}\right|_{g}^{2} \leq \sup _{t_{0} \leq s \leq t} \sup _{-\infty<v \leq 0}\left|X^{n}(s+v)\right|^{2} \leq \sup _{-\infty<r \leq t}\left|X^{n}(r)\right|^{2} \leq|\xi|_{g}^{2}+\sup _{t_{0} \leq r \leq t}\left|X^{n}(r)\right|^{2} .
$$

Again, noting that

$$
\max _{1 \leq n \leq k} E\left|X_{s}^{n-1}\right|_{g}^{2} \leq \max \left\{E|\xi|_{g}^{2}, \max _{1 \leq n \leq k} E\left|X_{s}^{n}\right|_{g}^{2}\right\} \leq E|\xi|_{g}^{2}+\max _{1 \leq n \leq k} E\left|X_{s}^{n}\right|_{g}^{2}
$$

for any $k \geq 1$.

By (7), (8) and Doob's martingale inequality, (6) becomes

$$
\begin{aligned}
\max _{1 \leq n \leq k} E\left|X^{n}(t)\right|^{2} \leq & z_{1}+12 K_{0} \max _{1 \leq n \leq k} E\left(\sup _{t_{0} \leq s \leq t}\left|X_{s}^{n}\right|_{g}^{2}\right)+8 b\left(T-t_{0}+1\right) \int_{t_{0}}^{t} \max _{1 \leq n \leq k} E\left|X_{s}^{n-1}\right|_{g}^{2} \mathrm{~d} s \\
\leq & z_{2}+12 K_{0} \max _{1 \leq n \leq k} E\left(|\xi|_{g}^{2}+\sup _{t_{0} \leq s \leq t}\left|X^{n}(s)\right|^{2}\right) \\
& +8 b\left(T-t_{0}+1\right) \int_{t_{0}}^{t} \max _{1 \leq n \leq k} E\left|X_{s}^{n}\right|_{g}^{2} \mathrm{~d} s \\
\leq & z_{2}+12 K_{0} \max _{1 \leq n \leq k} E\left(|\xi|_{g}^{2}+\sup _{t_{0} \leq s \leq t}\left|X^{n}(s)\right|^{2}\right) \\
& +8 b\left(T-t_{0}+1\right) \int_{t_{0}}^{t} \max _{1 \leq n \leq k} E\left(\sup _{t_{0} \leq r \leq s}\left|X_{r}^{n}\right|_{g}^{2}\right) \mathrm{d} s \\
\leq & z_{3}+12 K_{0} \max _{1 \leq n \leq k} E\left(\sup _{t_{0} \leq s \leq t}\left|X^{n}(s)\right|^{2}\right) \\
& +8 b\left(T-t_{0}+1\right) \int_{t_{0}}^{t} \max _{1 \leq n \leq k} E\left(\sup _{t_{0} \leq r \leq s}\left|X^{n}(r)\right|^{2}\right) \mathrm{d} s \\
\leq & z_{3}+48 K_{0} \max _{1 \leq n \leq k} E\left|X^{n}(t)\right|^{2} \\
& +32 b\left(T-t_{0}+1\right) \int_{t_{0}}^{t} \max _{1 \leq n \leq k} E\left|X^{n}(s)\right|^{2} \mathrm{~d} s,
\end{aligned}
$$

where $z_{2}=z_{1}+8 b\left(T-t_{0}+1\right)\left(T-t_{0}\right) E|\xi|_{g}^{2}, z_{3}=z_{2}+\left(12 K_{0}+8 b\left(T-t_{0}+1\right)\left(T-t_{0}\right)\right) E|\xi|_{g}^{2}$. Consequently,

$$
\max _{1 \leq n \leq k} E\left|X^{n}(t)\right|^{2} \leq \frac{z_{3}}{1-48 K_{0}}+\frac{32 b\left(T-t_{0}+1\right)}{1-48 K_{0}} \int_{t_{0}}^{t} \max _{1 \leq n \leq k} E\left|X^{n}(s)\right|^{2} \mathrm{~d} s .
$$

Let

$$
\alpha=\frac{z_{3}}{1-48 K_{0}}, \quad \beta=\frac{32 b\left(T-t_{0}+1\right)}{1-48 K_{0}},
$$


since $k$ is arbitrary, by means of Gronwall's inequality, we derive that

$$
E\left|X^{n}(t)\right|^{2} \leq \alpha e^{\beta\left(T-t_{0}\right)}:=\tilde{I}
$$

On the other hand, by the elementary inequality $(a+b+c)^{2} \leq 3\left(a^{2}+b^{2}+c^{2}\right)$, one gets

$$
\begin{aligned}
E\left|X^{n+m}(t)-X^{n}(t)\right|^{2} \leq & 3 E\left|D\left(t, X_{t}^{n+m}\right)-D\left(t, X_{t}^{n}\right)\right|^{2} \\
& +3 E\left|\int_{t_{0}}^{t}\left(f\left(s, X_{s}^{n+m-1}\right)-f\left(s, X_{s}^{n-1}\right)\right) \mathrm{d} s\right|^{2} \\
& +3 E\left|\int_{t_{0}}^{t}\left(h\left(s, X_{s}^{n+m-1}\right)-h\left(s, X_{s}^{n-1}\right)\right) \mathrm{d} W(s)\right|^{2} .
\end{aligned}
$$

From (B1), (B2), (B3), Hölder's inequality and Jensen inequality $E(\kappa(x)) \leq \kappa(E(x))$ for the concave function $\kappa(\cdot)$, it yields that

$$
\begin{aligned}
E\left(\sup _{t_{0} \leq s \leq t}\left|X^{n+m}(s)-X^{n}(s)\right|^{2}\right) \leq & 3 K_{0} E\left|X_{t}^{n+m}-X_{t}^{n}\right|_{g}^{2} \\
& +3\left(T-t_{0}+1\right) E \int_{t_{0}}^{t} \kappa\left(\left|X_{s}^{n+m-1}-X_{s}^{n-1}\right|_{g}^{2}\right) \mathrm{d} s \\
\leq & 3 K_{0} E\left(\sup _{t_{0} \leq r \leq t}\left|X^{n+m}(r)-X^{n}(r)\right|^{2}\right)+3\left(T-t_{0}+1\right) \\
& \times \int_{t_{0}}^{t} \kappa\left(E\left(\sup _{t_{0} \leq r \leq s}\left|X^{n+m-1}(r)-X^{n-1}(r)\right|^{2}\right)\right) \mathrm{d} s
\end{aligned}
$$

that is,

$$
E\left(\sup _{t_{0} \leq s \leq t}\left|X^{n+m}(s)-X^{n}(s)\right|^{2}\right) \leq \tilde{I}_{1} \int_{t_{0}}^{t} \kappa\left(E\left(\sup _{t_{0} \leq r \leq s}\left|X^{n+m-1}(r)-X^{n-1}(r)\right|^{2}\right)\right) \mathrm{d} s,
$$

where $\tilde{I}_{1}=\frac{3\left(T-t_{0}+1\right)}{1-3 K_{0}}$. From (11), (14) can be simplified as

$$
E\left(\sup _{t_{0} \leq s \leq t}\left|X^{n+m}(s)-X^{n}(s)\right|^{2}\right) \leq \tilde{I}_{1} \int_{t_{0}}^{t} \kappa(4 \tilde{I}) \mathrm{d} s \leq\left(T-t_{0}\right) \tilde{I}_{1} \kappa(4 \tilde{I}),
$$

where $\tilde{I}_{2}=\tilde{I}_{1} \kappa(4 \tilde{I})$. The proof is complete.

For all $t \in\left[t_{0}, T\right]$, define

$$
\psi_{1}(t)=\tilde{I}_{2}\left(t-t_{0}\right)
$$

For $n, m \geq 1$, define the recursive function

$$
\begin{aligned}
& \psi_{n+1}(t)=\tilde{I}_{1} \int_{t_{0}}^{t} \kappa\left(\psi_{n}(s)\right) \mathrm{d} s, \\
& \psi_{n, m}(t)=E\left(\sup _{t_{0} \leq r \leq t}\left|X^{n+m}(r)-X^{n}(r)\right|^{2}\right) .
\end{aligned}
$$


We choose $T_{1} \in\left[t_{0}, T\right]$ such that

$$
\tilde{I}_{1} \kappa\left(\tilde{I}_{2}\left(t-t_{0}\right)\right) \leq \tilde{I}_{2} \quad \text { for all } t \in\left[t_{0}, T_{1}\right]
$$

In fact, from the monotonicity of a concave function $\kappa(\cdot)$ and the relationship between $\tilde{I}$, $\tilde{I}_{1}$ and $\tilde{I}_{2}$, (19) becomes $\tilde{I}_{2}\left(t-t_{0}\right) \leq 4 \tilde{I}$, that is,

$$
t \leq \frac{4 \tilde{I}}{\tilde{I}_{2}}+t_{0}=\frac{4 \tilde{I}}{\tilde{I}_{1} \kappa(4 \tilde{I})}+t_{0}=\frac{1-3 K_{0}}{3\left(T-t_{0}+1\right)} \times \frac{4 \tilde{I}}{\kappa(4 \tilde{I})}+t_{0}
$$

From (5), and noting the fact that $\frac{x}{\kappa(x)} \geq \frac{1}{b}\left(1-\frac{a}{a+b x}\right)$, we can get

$$
t_{0} \leq t \leq \frac{1-3 K_{0}}{3 b\left(T-t_{0}+1\right)}+t_{0}=\frac{1-3 K_{0}}{3\left(T-t_{0}+1\right) \kappa^{\prime}\left(X_{T^{\prime \prime}}\right)}+t_{0}:=T_{1}
$$

Lemma 8 There exists a positive $T_{1} \in\left[t_{0}, T\right]$ such that

$$
0 \leq \psi_{n, m}(t) \leq \psi_{n}(t) \leq \psi_{n-1}(t) \leq \cdots \leq \psi_{1}(t)
$$

for all $t_{0} \leq t \leq T_{1}, n, m \geq 1$.

Proof According to the definition of the function $\psi(\cdot)$, we have

$$
\begin{aligned}
\psi_{1, m}(t) & =E\left(\sup _{t_{0} \leq s \leq t}\left|X^{1+m}(s)-X^{1}(s)\right|^{2}\right) \leq \tilde{I}_{2}\left(t-t_{0}\right)=\psi_{1}(t), \\
\psi_{2, m}(t) & =E\left(\sup _{t_{0} \leq s \leq t}\left|X^{2+m}(s)-X^{2}(s)\right|^{2}\right) \\
& \leq \tilde{I}_{1} \int_{t_{0}}^{t} \kappa\left(E\left(\sup _{t_{0} \leq r \leq s}\left|X^{1+m}(r)-X^{1}(r)\right|^{2}\right)\right) \mathrm{d} s \\
& \leq \tilde{I}_{1} \int_{t_{0}}^{t} \kappa\left(\psi_{1}(s)\right) \mathrm{d} s=\psi_{2}(t) .
\end{aligned}
$$

From (19), we also have

$$
\psi_{2}(t)=\tilde{I}_{1} \int_{t_{0}}^{t} \kappa\left(\psi_{1}(s)\right) \mathrm{d} s \leq \int_{t_{0}}^{t} \tilde{I}_{1} \kappa\left(\tilde{I}_{2}\left(t-t_{0}\right)\right) \mathrm{d} s \leq \tilde{I}_{2}\left(t-t_{0}\right)=\psi_{1}(t) .
$$

Thus we derive that $\psi_{2, m}(t) \leq \psi_{2}(t) \leq \psi_{1}(t)$ for all $t \in\left[t_{0}, T_{1}\right]$.

By induction, assume that $\psi_{n, m}(t) \leq \psi_{n}(t) \leq \psi_{n-1}(t) \leq \cdots \leq \psi_{1}(t), t_{0} \leq t \leq T_{1}$ holds for some $n \geq 1$. Now we check Lemma 8 is valid for $n+1$. In fact,

$$
\begin{aligned}
\psi_{n+1, m}(t) & =E\left(\sup _{t_{0} \leq r \leq t}\left|X^{n+m+1}(r)-X^{n+1}(r)\right|^{2}\right) \\
& \leq \tilde{I}_{1} \int_{t_{0}}^{t} \kappa\left(E\left(\sup _{t_{0} \leq r \leq s}\left|X^{n+m}(r)-X^{n}(r)\right|^{2}\right)\right) \mathrm{d} s=\tilde{I}_{1} \int_{t_{0}}^{t} \kappa\left(\psi_{n, m}(s)\right) \mathrm{d} s \\
& \leq \tilde{I}_{1} \int_{t_{0}}^{t} \kappa\left(\psi_{n}(s)\right) \mathrm{d} s=\psi_{n+1}(t) .
\end{aligned}
$$


From (17), one gets that

$$
\psi_{n+1}(t)=\tilde{I}_{1} \int_{t_{0}}^{t} \kappa\left(\psi_{n}(s)\right) \mathrm{d} s \leq \tilde{I}_{1} \int_{t_{0}}^{t} \kappa\left(\psi_{n-1}(s)\right) \mathrm{d} s=\psi_{n}(t)
$$

The proof is complete.

Prooffor the uniqueness of Theorem 1 Let $X(t)$ and $\bar{X}(t)$ be any two solutions of system (1) with the initial data (2). Thus, we have

$$
\begin{aligned}
|X(t)-\bar{X}(t)| \leq & \left|D\left(t, X_{t}\right)-D\left(t, \bar{X}_{t}\right)\right|+\int_{t_{0}}^{t}\left|f\left(s, X_{s}\right)-f\left(s, \bar{X}_{s}\right)\right| \mathrm{d} s \\
& +\left|\int_{t_{0}}^{t} h\left(s, X_{s}\right)-h\left(s, \bar{X}_{s}\right) \mathrm{d} W(s)\right| .
\end{aligned}
$$

By the elementary inequality $(a+b+c)^{2} \leq 3\left(a^{2}+b^{2}+c^{2}\right)$, Hölder's inequality and Lemma 4, one can find that

$$
\begin{aligned}
E|X(t)-\bar{X}(t)|^{2} \leq & 3 K_{0} E\left(\left|X_{t}-\bar{X}_{t}\right|_{g}^{2}\right)+3\left(t-t_{0}\right) E \int_{t_{0}}^{t}\left|f\left(s, X_{s}\right)-f\left(s, \bar{X}_{s}\right)\right|^{2} \mathrm{~d} s \\
& +3 E \int_{t_{0}}^{t}\left|h\left(s, X_{s}\right)-h\left(s, \bar{X}_{s}\right)\right|^{2} \mathrm{~d} s .
\end{aligned}
$$

From (B1), (B2), the definition of the norm at phase space $C_{g}$ and (7), then we have

$$
\begin{aligned}
E\left(\sup _{t_{0} \leq s \leq t}|X(s)-\bar{X}(s)|^{2}\right) \leq & 3 K_{0} E\left(\sup _{t_{0} \leq s \leq t}|X(s)-\bar{X}(s)|^{2}\right) \\
& +3\left(T-t_{0}+1\right) \int_{t_{0}}^{t} \kappa\left(E\left(\sup _{t_{0} \leq r \leq s}|X(r)-\bar{X}(r)|^{2}\right)\right) \mathrm{d} s,
\end{aligned}
$$

therefore

$$
E\left(\sup _{t_{0} \leq s \leq t}|X(s)-\bar{X}(s)|^{2}\right) \leq \frac{3\left(T-t_{0}+1\right)}{1-3 K_{0}} \int_{t_{0}}^{t} \kappa\left(E\left(\sup _{t_{0} \leq r \leq s}|X(r)-\bar{X}(r)|^{2}\right)\right) \mathrm{d} s .
$$

Lemma 5 and Lemma 6 yield $E\left(\sup _{t_{0} \leq s \leq t}|X(s)-\bar{X}(s)|^{2}\right)=0, t_{0} \leq t \leq T$. The proof of uniqueness is complete.

Proof for the existence of Theorem 1 Note that $\psi_{n}(t)$ is continuous on $\left[t_{0}, T_{1}\right]$. For each $n \geq 1, \psi_{n}(t)$ is decreasing on $\left[t_{0}, T_{1}\right]$, and for each $t, \psi_{n}(t)$ is also a decreasing sequence. From the dominated convergence theorem, we can define the function $\psi(t)$ as

$$
\psi(t)=\lim _{n \rightarrow \infty} \psi_{n}(t)=\lim _{n \rightarrow \infty} \tilde{I}_{1} \int_{t_{0}}^{t} \kappa\left(\psi_{n-1}(t)\right) \mathrm{d} s=\tilde{I}_{1} \int_{t_{0}}^{t} \kappa(\psi(s)) \mathrm{d} s, \quad t_{0} \leq t \leq T_{1} .
$$

Thus

$$
\psi(t) \leq \psi(0)+\tilde{I}_{1} \int_{t_{0}}^{t} \kappa(\psi(s)) \mathrm{d} s
$$

For all $t_{0} \leq t \leq T_{1}$, Lemma 5 and Lemma 6 imply that $\psi(t)=0$. 
By Lemma 8, for $t_{0} \leq t \leq T_{1}$, we have $\psi_{n, m}(t) \leq \psi_{n}(t) \rightarrow 0$ as $n \rightarrow \infty$, that is, $E \mid X^{n+m}(t)-$ $\left.X^{n}(t)\right|^{2} \rightarrow 0$ as $n \rightarrow \infty$. From the completeness of $L^{2}$, the assumptions (B1), (B2), (B3) and the property of the function to $\kappa(\cdot)$, it then follows that for all $t \in\left[t_{0}, T_{1}\right]$,

$$
D\left(s, X_{s}^{n}\right) \stackrel{L^{2}}{\rightarrow} D\left(s, X_{s}\right), \quad f\left(s, X_{s}^{n}\right) \stackrel{L^{2}}{\rightarrow} f\left(s, X_{s}\right), \quad h\left(s, X_{s}^{n}\right) \stackrel{L^{2}}{\rightarrow} h\left(s, X_{s}\right) \quad \text { as } n \rightarrow \infty .
$$

Then, for all $t \in\left[t_{0}, T_{1}\right]$,

$$
\begin{aligned}
\lim _{n \rightarrow \infty} X^{n}(t)= & \xi(0)+\lim _{n \rightarrow \infty} D\left(t, X_{t}^{n}\right)-D\left(t_{0}, \xi\right)+\lim _{n \rightarrow \infty} \int_{t_{0}}^{t} f\left(s, X_{s}^{n-1}\right) \mathrm{d} s \\
& +\lim _{n \rightarrow \infty} \int_{t_{0}}^{t} h\left(s, X_{s}^{n-1}\right) \mathrm{d} W(s),
\end{aligned}
$$

that is,

$$
X(t)=\xi(0)+D\left(t, X_{t}\right)-D\left(t_{0}, \xi\right)+\int_{t_{0}}^{t} f\left(s, X_{s}\right) \mathrm{d} s+\int_{t_{0}}^{t} h\left(s, X_{s}\right) \mathrm{d} W(s)
$$

which demonstrates that $X(t)$ is one solution of system (1) with initial data (2) on $\left[t_{0}, T_{1}\right]$, where $T_{1} \in\left[t_{0}, T\right]$. By iteration, the existence of solutions to system (1) on $\left[t_{0}, T\right]$ can be obtained. The proof is complete.

From Theorem 1, the existence and uniqueness of solutions to system (1) are defined on a finite interval $\left[t_{0}, T\right]$. If all additions of the existence and uniqueness theorem are satisfied on every finite subinterval of $\left[t_{0},+\infty\right)$, then system (1) will have a unique solution $X(t)$ on the entire interval $(-\infty,+\infty)$. So, we have the following corollary.

Corollary 1 Assume that for each real number $T>t_{0}$, there exist positive constants $K_{T}$ and $\bar{K}_{T}$ such that for all $t \in\left[t_{0}, T\right]$ and all $\varphi, \psi \in C_{g}$, it follows that

$$
\begin{aligned}
& |f(t, \varphi)-f(t, \psi)|^{2} \vee|h(t, \varphi)-h(t, \psi)|^{2} \leq \kappa\left(|\varphi-\psi|_{g}^{2}\right), \\
& |f(t, 0)|^{2} \vee|h(t, 0)|^{2} \leq K_{T}, \\
& |D(t, \varphi)-D(t, \psi)|^{2} \leq \bar{K}_{T}|\varphi-\psi|_{g}^{2} \text { and }|D(t, 0)|^{2} \leq \bar{K}_{T},
\end{aligned}
$$

where $\kappa(\cdot)$ is defined in (B1). Then the system

$$
\mathrm{d}\left(X(t)-D\left(t, X_{t}\right)\right)=f\left(t, X_{t}\right) \mathrm{d} t+h\left(t, X_{t}\right) \mathrm{d} W(t), \quad t_{0} \in\left[t_{0},+\infty\right)
$$

has a unique global solution $X(t)$; moreover, $X(t) \in \mathcal{M}^{2}\left((-\infty,-\infty) ; \mathrm{R}^{d}\right)$.

The proof is similar to that in Theorem 1, we omit it here.

\section{Continuous dependence of solutions with initial value}

In this section, we give the continuous dependence of solutions for system (1) with the initial data (2) by means of Lemma 5 and Lemma 6. 
Theorem 2 Let $X^{\xi}(t)$ and $Y^{\eta}(t)$ be two solutions of system (1) with initial data $\xi$ and $\eta$, respectively. Assume that (B1), (B2) and (B3) are satisfied if for all $\varepsilon>0$ and $t \in\left[t_{0}, T\right]$, there exists $\delta(\varepsilon)>0$ such that $E|\xi-\eta|_{g}^{2}<\delta(\varepsilon)$, then $E\left|X^{\xi}(t)-Y^{\eta}(t)\right|^{2} \leq \varepsilon$.

Proof For any $t \in\left[t_{0}, T\right]$, it follows that

$$
\begin{aligned}
& X(t)=\xi(0)+D\left(t, X_{t}\right)-D\left(t_{0}, \xi\right)+\int_{t_{0}}^{t} f\left(s, X_{s}\right) \mathrm{d} s+\int_{t_{0}}^{t} h\left(s, X_{s}\right) \mathrm{d} W(s) \quad \text { a.s., } \\
& Y(t)=\eta(0)+D\left(t, Y_{t}\right)-D\left(t_{0}, \eta\right)+\int_{t_{0}}^{t} f\left(s, Y_{s}\right) \mathrm{d} s+\int_{t_{0}}^{t} h\left(s, Y_{s}\right) \mathrm{d} W(s) \quad \text { a.s. }
\end{aligned}
$$

Then

$$
\begin{aligned}
X(t)-Y(t)= & \xi(0)-\eta(0)+D\left(t, X_{t}\right)-D\left(t, Y_{t}\right)+D\left(t_{0}, \eta\right)-D\left(t_{0}, \xi\right) \\
& +\int_{t_{0}}^{t}\left(f\left(s, X_{s}\right)-f\left(s, Y_{s}\right)\right) \mathrm{d} s+\int_{t_{0}}^{t}\left(h\left(s, X_{s}\right)-h\left(s, Y_{s}\right)\right) \mathrm{d} W(s) \quad \text { a.s. }
\end{aligned}
$$

By the same arguments, we get that

$$
\begin{aligned}
& E\left(\sup _{t_{0} \leq s \leq t}|X(s)-Y(s)|^{2}\right) \\
& \quad \leq \frac{5}{1-8 K_{0}} E|\xi-\eta|_{g}^{2}+\int_{t_{0}}^{t} \frac{4\left(T-t_{0}+1\right)}{1-8 K_{0}} \kappa\left(E\left(\sup _{t_{0} \leq r \leq s}|X(r)-Y(r)|^{2}\right)\right) \mathrm{d} s .
\end{aligned}
$$

Let $u_{0}=\frac{5}{1-8 K_{0}} E|\xi-\eta|_{g}^{2} \geq 0, u(t)=E\left(\sup _{t_{0} \leq s \leq t}|X(s)-Y(s)|^{2}\right), v(t)=\frac{4\left(T-t_{0}+1\right)}{1-8 K_{0}}$, for $\kappa(\cdot)$ is a concave continuous increasing function from $\mathrm{R}^{+}$to $\mathrm{R}^{+}$such that $\kappa(u)>0$ for $u>0, \kappa(0)=0$ and $\int_{0^{+}} \frac{1}{\kappa(u)} \mathrm{d} u=\infty$. Therefore, for any $\varepsilon>0, \varepsilon_{1}=\frac{1}{6} \varepsilon, K_{0}<\frac{1}{48}$, it yields that $\int_{u_{0}}^{\varepsilon_{1}} \frac{1}{\kappa(u)} \mathrm{d} u \geq$ $\int_{t_{0}}^{T} v(s) \mathrm{d} s$ as $u_{0} \leq \varepsilon_{1}$. From Lemma 5 and Lemma 6 , for any $t \in\left[t_{0}, T\right]$, the estimate $u(t) \leq \varepsilon_{1}$ holds, i.e., $E\left(\sup _{t_{0} \leq s \leq t}|X(s)-Y(s)|^{2}\right) \leq \varepsilon_{1}$. The proof is complete.

Corollary 2 Let $X^{\xi}(t)$ and $Y^{\eta}(t)$ be two solutions of system (1) with initial data $\xi$ and $\eta$, respectively. Assume that (B1), (B2) and (B3) are satisfied if for all $\varepsilon>0$ and $p \geq 2$, there exists $\delta(\varepsilon)>0$ such that $E|\xi-\eta|_{g}^{p}<\delta(\varepsilon)$, then $E\left|X^{\xi}(t)-Y^{\eta}(t)\right|^{p} \leq \varepsilon$ for $t \in\left[t_{0}, T\right]$.

\section{Competing interests}

The authors declare that they have no competing interests.

Authors' contributions

All authors conceived of the study and carried out the proof. All authors read and approved the final manuscript.

\section{Acknowledgements}

This research is supported by NNSF of China (11201075), the Natural Science Foundation of Fujian Province of China (2010J01005) and the Technology Innovation Platform Project of Fujian Province (No. 2009J1007). The authors are thankful to the referees for their careful reading of the manuscript and insightful comments.

Received: 12 February 2013 Accepted: 10 May 2013 Published: 28 May 2013

\section{References}

1. Hale, JK: Theory of Functional Differential Equations. Springer, New York (1977)

2. Wang, K, Fan, M: Phase Space Theory and Their Application of Functional Differential Equations. Science Press, Beijing (2009)

3. Hale, JK, Kato, J: Phase space for retarded equations with infinite delay. Funkc. Ekvacioj 21(1), 11-41 (1978) 
4. Schumacher, K: Existence and continuous dependence for functional-differential equations with unbounded delay. Arch. Ration. Mech. Anal. 67(4), 315-335 (1978)

5. Schumacher, K: Dynamical systems with memory on history-spaces with monotonic seminorms. J. Differ. Equ. 34(3), 440-463 (1979)

6. Corduneanu, C, Lakshmikantham, V: Equations with unbounded delay: a survey. Nonlinear Anal. 4(5), 831-877 (1980)

7. Mao, XR: Stochastic Differential Equations and Applications. Horwood, Chichester (1997)

8. Zheng, ZX: The development and application of functional differential equations. Adv. Math. 12(2), 94-112 (1983)

9. Friedman, A: Stochastic Differential Equations and Their Applications. Academic Press, San Diego (1976)

10. Wei, FY: The basic theory of stochastic functional differential equations with infinite delay. Dissertation, Northeast Normal University, China (2006)

11. Wei, FY, Wang, K: The existence and uniqueness of the solution for stochastic functional differential equations with infinite delay. J. Math. Anal. Appl. 331(1), 516-531 (2007)

12. $\mathrm{Xu}, \mathrm{DY}$, Yang, ZG, Huang, YM: Existence-uniqueness and continuation theorems for stochastic functional differential equations. J. Differ. Equ. 245(6), 1681-1703 (2008)

13. Yue, $\mathrm{CH}, \mathrm{Wu}$, J: Existence, uniqueness and stability of the solution to stochastic functional differential equations with infinite delay at phase space $C_{h}$. J. Univ. Sci. Technol. China 40(6), 583-589 (2010)

14. Su, H, Li, WX, Wang, K, Ding, XH: Stability analysis for stochastic neural network with infinite delay. Neurocomputing 74(10), 1535-1540 (2011)

15. Zhang, CM, Li, WX, Wang, K: Stability and boundedness of stochastic Volterra integrodifferential equations with infinite delay. J. Appl. Math. 2013, Article ID 320832 (2013)

16. Mao, XR: Asymptotic properties of neutral stochastic differential delay equations. Stoch. Int. J. Probab. Stoch. Process. 68(3/4), 273-295 (2000)

17. Zhou, SB, Xue, MG: The existence and uniqueness of the solutions for neutral stochastic functional differential equations with infinite delay. Math. Appl. 21(1), 75-83 (2008)

18. Ren, Y, Xia, NM: Existence, uniqueness and stability of the solutions to neutral stochastic functional differential equations with infinite delay. Appl. Math. Comput. 210(1), $72-79$ (2009)

19. $\mathrm{Xu}, \mathrm{Y}, \mathrm{Hu}, \mathrm{SG}$ : The existence and uniqueness of the solution for neutral stochastic functional differential equations with infinite delay in abstract space. Acta Appl. Math. 110(2), 627-638 (2010)

20. Chen, HB: The existence and uniqueness for the solution of neutral stochastic functional differential equations with infinite delay. J. Math. Res. Expo. 30(4), 589-598 (2010)

21. Arino, OA, Burton, TA, Haddock, JR: Periodic solutions to functional differential equations. Proc. R. Soc. Edinb. A 101, 253-271 (1985)

doi:10.1186/1687-1847-2013-151

Cite this article as: Wei and Cai: Existence, uniqueness and stability of the solution to neutral stochastic functional differential equations with infinite delay under non-Lipschitz conditions. Advances in Difference Equations 2013 2013:151.

\section{Submit your manuscript to a SpringerOpen ${ }^{\circ}$ journal and benefit from:}

- Convenient online submission

Rigorous peer review

- Immediate publication on acceptance

Open access: articles freely available online

- High visibility within the field

- Retaining the copyright to your article 